\title{
El uso de la información por los docentes de nivel secundaria en México
}

\author{
Angélica Guevara Villanueva* \\ Universidad Nacional Autónoma de México. Facultad de Filosofía y Letras \\ angelicamatem@gmail.com
}

Recibido: Julio 2015

Aceptado: Octubre 2015

Resumen: La siguiente investigación muestra los resultados que fueron obtenidos a través de la aplicación de una cédula de entrevista a la comunidad de docentes de nivel secundaria de la modalidad general que se encuentran adscritos al Distrito Federal con el objetivo de analizar los hábitos que han desarrollado en la búsqueda de la información que ha permitido ejercer su actividad docente y cumplir con las políticas educativas imperantes en materia de evaluación. Bajo este respecto, se hace mención de manera general acerca de los primeros estudios de usuarios en comunidades científicas y académicas y sobre las modalidades que integran al nivel de secundaria en México. Asimismo, se describen los elementos metodológicos que guiaron el proceso de la investigación que se llevó a cabo para poder entrevistar a los docentes. Finalmente, se presentan los resultados y conclusiones.

Palabras clave: Comportamiento en la búsqueda de información; Comportamiento informativo; Distrito Federal; México; Profesores de nivel secundaria; Profesores de enseñanza secundaria.

\section{The use of information by teachers of the secondary level in Mexico}

\begin{abstract}
The following research shows the results obtained through interviews to the members of a teachers' community in the secondary school level. These teachers are in charge of groups of students in Mexico City in schools of general type. The purpose of the research was the analysis of the habits in the research of information needed for their teaching and to comply with the official policies in education assessment. In this respect, we give a general mention about previous studies of this kind in scientific and academic communities, as well as in teachers' communities in schools of specific types in Mexico. We also describe the methodological elements which conducted the research process for a interviews the teachers. Finally we make a results and give the conclusions.
\end{abstract}

Keywords: Behavior in the search of information; High school level; Mexico City; Secondary school teachers; Seeking behavior information.

* Esta investigación forma parte de un estudio más extenso que se está realizando en los estudios de doctorado dentro del programa de posgrado en bibliotecología y estudios de la información en la Facultad de Filosofía y Letras de la Universidad Nacional Autónoma de México. 


\section{INTRODUCCIÓN}

Sin lugar a dudas, la comunidad de docentes de nivel secundaria como grupo social definido tiene una misión propia y fundamental dentro de la sociedad que exige de una capacitación, actualización y preparación profesional continua que permita adquirir las habilidades y competencias para poder ejercer con profesionalismo su actividad docente y cumplir con las políticas educativas imperantes en materia de evaluación.

Pero además de lo anterior, resalta un aspecto que figura como eje de acción para el cumplimiento de los requerimientos que los maestros de nivel secundaria deben de ser capaces de cubrir específicamente me refiero, al proceso de búsqueda, manejo y uso de la información que debe de estar incorporado en las actividades laborales, profesionales y académicas que realizan los profesores.

Es así, que partiendo de este escenario, esta investigación persigue describir, analizar e interpretar los hechos que ocurren en la práctica docente dentro y fuera del aula, para llegar a definir algunos rasgos personales del docente y los hábitos que han desarrollado en el proceso de búsqueda de la información. Sobre ese particular no se busca evaluar al docente, ni enjuiciar, sino más bien, entender que la existencia de factores internos y externos puede influir en la manera como se comportan hacia la búsqueda de la información ante la idea de que puedan alcanzar los niveles de exigencia que la sociedad reclama.

No obstante, que los primeros estudios de usuarios de la información comienzan a elaborarse a partir de la década de los veinte en distintas comunidades de investigadores en el ámbito norteamericano sobre algunos aspectos relacionados con las fuentes de información formales e informales así como, en el estudio de los hábitos de comunicación entre los científicos y la valoración del uso que se hacía de los sistemas y servicios de información principalmente en científicos experimentales y tecnólogos (Sanz,1993:158) y, más sólidamente, en el año de 1948 en la Royal Society Scientific Information Conference, donde Urquhart (1948) y Bernal (1948) reportaron sus descubrimientos de investigación basados en la distribución y el uso de la información científica y técnica, entre otros aspectos, (González, 2005: 41-42) es hasta finales de la década de los sesenta y principios de los setenta cuando se comienzan a elaborar algunas indagaciones con respecto a las necesidades de información y a los hábitos en la búsqueda de información dentro de las comunidades científicas de las ciencias sociales y humanísticas, donde la pedagogía como disciplina humanística, vino a ocupar un lugar de interés apareciendo de este modo, los primeros trabajos elaborados por Havelock (1967; 1969); Chorness, Rittenhouse y Heald (1968); Rittenhouse (1970) y Summers (1974).

Pese a esto, en México los profesores de secundaria siguen siendo un gremio poco estudiado dentro de la línea de investigación denominada estudios de usuarios de la información, prueba de ello, ha sido la realización hasta el momento de una indagación elaborada por Arriola y Butrón (2008) en donde se analiza el servicio que ofrece el personal bibliotecario, el ambiente físico, el uso de algunas 
fuentes de información, las herramientas tecnológicas y el equipamiento de dieciocho Centros de Maestros ${ }^{1}$ en el Distrito Federal. ${ }^{2}$

Por lo que esta investigación, representa un primer acercamiento para conocer y profundizar acerca del conocimiento de los profesores con el fin de disponer de elementos de juicio que conviden a la reflexión y participación de los profesionales de la información para proponer acciones dirigidas hacia el mejoramiento de las condiciones de organización, difusión y acceso a la información.

\section{LA EDUCACIÓN SECUNDARIA EN EL CONTEXTO DEL SISTEMA EDUCATIVO MEXICANO}

De manera general, la educación secundaria se concibe como un conjunto de estudios que sitúa entre la enseñanza primaria y la superior (de ahí su nombre de secundaria, intermedia, media, etcétera), ésta se había considerado hasta febrero del 2012 como la última parte de la enseñanza básica obligatoria, sin embargo, por decreto presidencial publicado en el Diario Oficial de la Federación (9 de febrero, 2012) hoy en día, constituye una etapa de la educación básica que junto con la primaria y el bachillerato contribuyen al desarrollo integral de la persona (Quiñones, 1992: 4).

De este modo, la ampliación de la matrícula en el nivel de secundaria se ha dado en el marco de la diversificación de modalidades educativas, de las cuales existen en la actualidad cinco: general, técnica, telesecundaria, para trabajadores y comunitaria. (Sandoval, 2008: 79) Pero, ¿qué es la modalidad educativa?

Se puede entender por modalidad educativa o de enseñanza como la forma en que diseña un programa escolar determinado. La modalidad busca adecuarse a las necesidades de grupos bien diferenciados en la sociedad y, por ello, afecta a todos los elementos del proceso de enseñanza-aprendizaje, desde los objetivos, pasando por contenidos y técnicas de enseñanza, materiales didácticos y sistemas de evaluación del aprendizaje, hasta los criterios de promoción a grados superiores. (López, Morales y Silva, 2005: 44)

En este sentido, las diversas modalidades educativas pretenden objetivos de formación comunes pues ofrecen a sus estudiantes un mismo currículo, no obstante, guardan algunas diferencias respecto de su estructura y forma de

\footnotetext{
${ }^{1}$ Los Centros de Maestros son espacios destinados principalmente a proporcionar asesoría a los docentes inscritos en los cursos de actualización mediante diversas instalaciones y servicios como biblioteca, sala de lectura, servicio para la recepción, grabación y reproducción de audio $\mathrm{y}$ video, entre otros.

${ }^{2}$ La Ciudad de México o también llamada Distrito Federal es la capital y sede de los poderes federales de los Estados Unidos Mexicanos se encuentra dividida en 16 delegaciones. Cada delegación está integrada por pueblos, barrios y colonias.
} 
organización. Por ejemplo, en la modalidad general cada asignatura suele ser impartida por un profesor distinto especializado en la disciplina operando en turno matutino o vespertino con sostenimiento público y en donde existen talleres y laboratorios para que los estudiantes puedan desarrollar sus habilidades y aprendizajes mediante la práctica, (México. SEP, 2012a: 3) se brinda a lo largo y ancho del territorio mexicano a través de la Secretaría de Educación Pública (SEP) quien es la responsable de la planeación y evaluación del Sistema Educativo Nacional. Asimismo, es la modalidad en donde se encuentra el mayor número de docentes y alumnos. ${ }^{3}$

Dada su importancia y características que representa en la sociedad mexicana, para fines de esta investigación se ha optado por seleccionar a los profesores que se encuentran dentro de esta modalidad y que se localizan en el Distrito Federal por ser la entidad que concentra el mayor número de docentes en activo. Bajo este criterio los objetivos centrales que se pretenden cumplir son los siguientes:

- Establecer las estrategias y los procedimientos de búsqueda de información que realiza la comunidad de profesores de secundaria de la modalidad general en el Distrito Federal.

- Determinar los factores (internos y/o externos) que influyen en su comportamiento informativo de la comunidad de profesores de secundaria de la modalidad general en el Distrito Federal

Ahora bien considerando que la información es un elemento indispensable para la preparación, actualización, capacitación y superación de cualquier profesionista y, más aún, para la comunidad de profesores de nivel secundaria que se dedican a la transmisión de conocimientos, a continuación se expresan las siguientes hipótesis:

- El procedimiento de búsqueda de información que los profesores han desarrollado se dirige principalmente hacia el uso de los materiales impresos, dejando de lado el empleo de Internet y otros medios electrónicos. Asimismo, existe una mayor tendencia a recurrir hacia los Centros de Maestros que a la comunicación entre colegas.

- Las fuentes y recursos electrónicos son utilizados con mayor frecuencia por los profesores jóvenes ${ }^{4}$ en comparación con aquellos colegas que tienen una edad mayor.

${ }^{3}$ Ciclo escolar 2011-2012. Alumnos: 3,127,675. Profesores: 219,021. (México. Secretaría de Educación Pública. Sistema Educativo de los Estados Unidos Mexicanos, principales cifras, ciclo escolar 2000-2001; 2006-2007 y 2011-2012. México: SEP, Dirección General de Planeación y Programación.)

${ }^{4}$ De acuerdo con Instituto Mexicano de la Juventud se tipifica como joven a aquellas personas que su edad fluctúa entre los 12 a los 29 años de edad. (2014). 


\section{METODOLOGÍA}

Debido a la dimensión de los profesores de secundaria de la modalidad seleccionada que suman un total de 20,998 y a su distribución geográfica dentro las 16 delegaciones que conforman al Distrito Federal ${ }^{5}$ la investigación se guio a través de la utilización de un método directo y la investigación de campo. Además, se tomó la decisión de recurrir a la técnica de la encuesta y por instrumento la elaboración de una cédula de entrevista. También, fue necesario obtener una muestra probabilística de la población (Martínez, 2002) y realizar una estratificación para tener representatividad en cada una de las delegaciones (Hernández; Fernández y Baptista, 2010). No obstante a estos datos, a su vez, se consideró la visita a una escuela por cada sujeto de análisis con la finalidad de no tener sesgos en la investigación. De esta manera, a través de la tabla 1 se puede observar que del total de la población la muestra estuvo representada por 270 educadores elegidos con base en los criterios de inclusión previamente determinados. ${ }^{6}$

Tabla 1. Cantidad de profesores que integraron la muestra en cada delegación

\begin{tabular}{|c|c|c|}
\hline Delegación Política & $\begin{array}{c}\text { Personal docente en Distrito } \\
\text { Federal }\end{array}$ & $\begin{array}{c}\text { Cantidad de profesores que forman } \\
\text { parte del estudio }\end{array}$ \\
\hline Álvaro Obregón & 881 & 11 \\
\hline Azcapotzalco & 1270 & 16 \\
\hline Benito Juárez & 866 & 11 \\
\hline Coyoacán & 1224 & 16 \\
\hline Cuajimalpa de Morelos & 275 & 4 \\
\hline Cuauhtémoc & 3000 & 39 \\
\hline Gustavo A. Madero & 3163 & 41 \\
\hline Iztacalco & 1168 & 15 \\
\hline Iztapalapa & 4135 & 53 \\
\hline Magdalena Contreras & 400 & 5 \\
\hline Miguel Hidalgo & 1062 & 14 \\
\hline Milpa Alta & 243 & 3 \\
\hline Tláhuac & 540 & 7 \\
\hline Tlalpan & 725 & 9 \\
\hline Venustiano Carranza & 1245 & 16 \\
\hline Xochimilco & 801 & 10 \\
\hline Total & 20,998 & 270 \\
\hline
\end{tabular}

Fuente: Elaboración propia

http://www.imjuventud.gob.mx/quienes-somos-imjuve-31.html [Consulta: 12/10/2014]

${ }^{5}$ Con base a la nómina de la plantilla docente de nivel secundaria correspondiente a la quincena 19, (octubre) 2012 y a las estadísticas de secundaria del ciclo escolar 2011-2012 de la Secretaría de Educación Pública.

${ }^{6}$ Los criterios fueron: poseer dos años de antigüedad como mínimo, contar con una plaza de base o interinato sin titular, tener el grado académico correspondiente al nivel educativo, desempeñar las funciones propias de la tres modalidades general y encontrarse en activo. 


\section{ANÁLISIS DE RESULTADOS}

Antes de presentar los resultados con respecto a su comportamiento en la búsqueda de la información de la comunidad docente es importante indicar que la cédula de entrevista inició con una serie de preguntas personales con la intención de determinar los factores (internos y/o externos) y las variables que inciden en su comportamiento informativo. (véase apéndice) De este modo, para establecer la correlación entre las variables se utilizó la escala de valoración del Coeficiente de Pearson $^{7}$ y la Prueba de significación Ji cuadrada (Rojas, 2000). Por ello, a continuación se dan a conocer los siguientes datos:

- $\quad$ El $60.0 \%$ perteneció al sexo de las mujeres (véase tabla 2)

- $\quad$ El $49.6 \%$ se encontró bajo un régimen conyugal casado (véase tabla 2)

- El 76.3\% manifestó tener dependientes económicos (véase tabla 2)

- El 24.8\% tuvo en promedio una edad de 31 a 40 años (véase tabla 3)

- El 31.1\% tuvo una antigüedad entre 11 a 15 años (véase tabla 3)

- El 50.7\% laboró de un horario de 31 a 40 horas (véase tabla 4)

- El 67.0\% tuvo un nivel de estudio de licenciatura (véase tabla 4)

- El 67.0\% no se encontró estudiando (véase tabla 4)

Tabla 2. Género, Estado civil y Dependientes económicos

\begin{tabular}{|c|c|c|c|c|c|c|c|c|}
\hline \multicolumn{2}{|c|}{ Sexo (\%) } & & \multicolumn{3}{c|}{ Estado Civil (\%) } & & \multicolumn{2}{c|}{$\begin{array}{c}\text { Dependientes } \\
\text { Económicos (\%) }\end{array}$} \\
\hline Mujeres & Hombres & & Soltera(o) & Casada(o) & Otro & & Si & No \\
\hline 60.0 & 40.0 & & 34.1 & 49.6 & 16.3 & & 76.3 & 23.7 \\
\hline
\end{tabular}

Fuente: Elaboración propia

Tabla 3. Edad y Antigüedad laboral

\begin{tabular}{|l|c|c|c|c|c|c|c|c|c|c|c|c|c|}
\hline \multicolumn{7}{|c|}{ Edad (\%) } & \multicolumn{5}{|c|}{ Antigüedad Laboral (\%) } \\
\hline $20-$ & $26-$ & $31-$ & $36-$ & $41-$ & $46-$ & $>$ a & $<$ de & $6-$ & $11-$ & $16-$ & $21-$ & $>$ a \\
25 & 30 & 35 & 40 & 45 & 50 & 50 & 5 & 10 & 15 & 20 & 25 & 26 \\
\hline 7.4 & 8.5 & 12.6 & 24.8 & 20.7 & 7.8 & 18.1 & 13.7 & 24.4 & 31.1 & 13.7 & 10.4 & 6.7 \\
\hline
\end{tabular}

Fuente: Elaboración propia

${ }^{7}$ Escala de valoración del coeficiente de Pearson:

Valor del coeficiente

Menos de .25

De .26 a .45

De .46 a .55

De .56 a .75

De .76 en adelante
Magnitud de la asociación o correlación

Baja

Media baja

Media

Media alta

Alta 
Tabla 4. Horas laborables, Nivel de estudio y Se encontró estudiando

\begin{tabular}{|c|c|c|c|c|c|c|c|c|}
\hline \multicolumn{4}{|c|}{ Horas laborales (\%) } & \multicolumn{3}{|c|}{ Nivel de estudio (\%) } & \multicolumn{2}{|c|}{ Estudia (\%) } \\
\hline$<$ de 10 & $10-20$ & $21-30$ & $31-40$ & Licenciatura & Maestría & Otro & $\mathrm{Si}$ & No \\
\hline 8.2 & 18.5 & 22.6 & 50.7 & 67.0 & 11.5 & 21.5 & 33.0 & 67.0 \\
\hline
\end{tabular}

Fuente: Elaboración propia

En lo que corresponde al uso de la información dentro de la comunidad académica las respuestas obtenidas manifestaron que la acción que realizaron en un primer momento para llevar a cabo sus actividades de capacitación, actualización y preparación profesional fue recurrir hacia la consulta de Internet (64.4\%), porcentaje que se puede observar en la siguiente tabla 5 en donde se muestra que 174 docentes respondieron usar éste recurso. En segundo lugar, se encontró que consultaron a sus colegas (21.1\%) y en tercer sitio, buscaron en su colección particular (7.8\%). En cantidades menores se aprecia su asistencia a la biblioteca (3.7\%), la confianza en su experiencia y conocimientos personales (1.1\%) y recurrir a eventos (1.5\%). Haciendo un análisis de correlación entre la variable edad y recurso informativo más utilizado se obtuvo que $C=0.14$, una magnitud de correlación baja, que permite corroborar que la edad no es un factor que influya en la preferencia de los docentes por emplear el Internet. Circunstancia que ratifica que el desarrollo constante y el uso de éste recurso ha sido un medio indispensable que ha favorecido la labor académica y profesional de las profesoras y profesores del nivel de secundaria.

Tabla 5. Recurso informativo al que acuden

\begin{tabular}{|l|c||c||}
\hline \multicolumn{1}{|c|}{ Recurso informativo } & $\begin{array}{c}\text { No. de } \\
\text { docentes }\end{array}$ & Porcentaje \\
\hline \hline Preguntar a sus colegas & 57 & $21.1 \%$ \\
\hline \hline Consultar Internet & 174 & $64.4 \%$ \\
\hline \hline Consultar su colección particular & 21 & $7.8 \%$ \\
\hline $\begin{array}{l}\text { Asistir a la biblioteca (Biblioteca escolar, Biblioteca } \\
\text { Pública, Centro de Maestros) }\end{array}$ & 10 & $3.7 \%$ \\
\hline Confiar en su experiencia y conocimientos personales & 3 & $1.1 \%$ \\
\hline $\begin{array}{l}\text { Recurrir a eventos (congresos, reuniones, talleres, } \\
\text { encuentros, etc.) }\end{array}$ & 4 & $1.5 \%$ \\
\hline \hline Asistir a librerías & 1 & $0.4 \%$ \\
\hline \hline
\end{tabular}

Fuente: Elaboración propia

Otro aspecto que se indagó en los docentes fue la razón del porqué recurrieron a realizar esta primera acción que indicaron en la pregunta anterior -el consultar Internet-. Bajo esta interrogante, las cifras orientaron que suelen consultarlo básicamente por su accesibilidad (75.5\%) (véase figura 1), en un segundo y tercer momento, por su confiabilidad (15.2\%), y rapidez (9.3\%). 
Figura 1. Razón de recurrir al uso de Internet

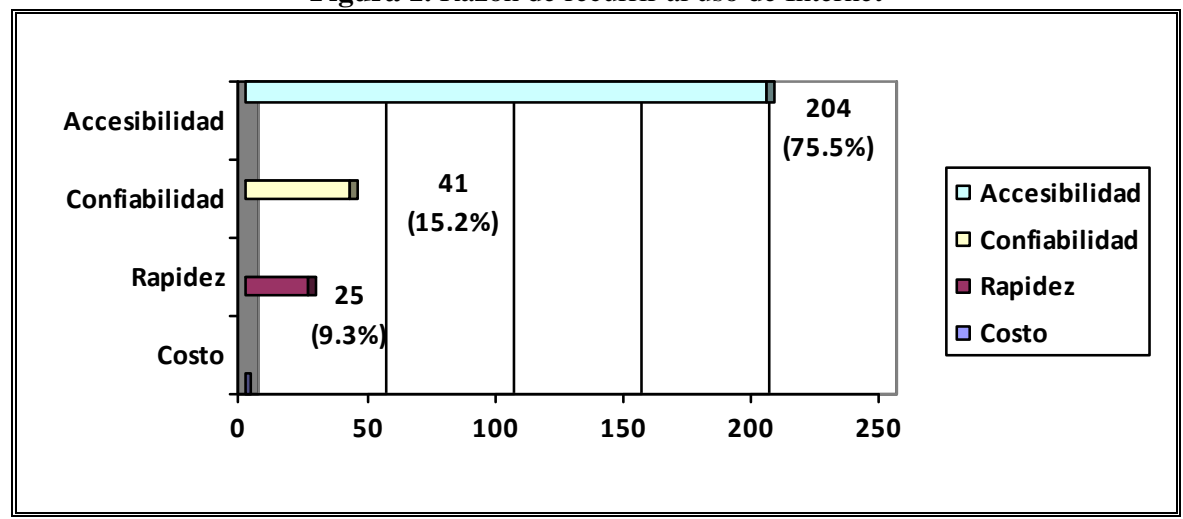

Fuente: Elaboración propia

Por lo que respecta, al uso de las fuentes informativas que utilizaron en mayor medida la comunidad de profesores las cantidades siguientes (véase tabla 6) evidenciaron que los materiales más empleados fueron los libros de carácter general (50.7\%), seguido de los libros de texto del alumno (18.5\%) y los libros para el maestro (16.4\%). ${ }^{8}$ Aunque también hay que señalar que, algunos otros mencionaron utilizar los avances programáticos (6.3\%), la guía para el maestro (4.8\%), y en menor medida, los materiales no impresos (1.8\%), las notas de cursos, seminarios y ponencias (1.1\%), y los ficheros de actividades didácticas (0.4\%).

Tabla 6. Fuente informativa más consultada

\begin{tabular}{|l|c||c||}
\hline \multicolumn{1}{|c|}{ Fuentes informativas } & No. de docentes & Porcentaje \\
\hline \hline Libros de carácter general & 137 & $50.7 \%$ \\
\hline \hline Libros para el maestro & 44 & $16.4 \%$ \\
\hline \hline Libros “guía para el maestro” & 13 & $4.8 \%$ \\
\hline \hline Libros de texto del alumno & 50 & $18.5 \%$ \\
\hline \hline Ficheros de actividades didácticas & 1 & $0.4 \%$ \\
\hline Avances programáticos & 3 & $6.3 \%$ \\
\hline \hline $\begin{array}{l}\text { Notas de cursos, seminarios, ponencias, talleres, reuniones, } \\
\text { congresos etc. }\end{array}$ & 5 & $1.1 \%$ \\
\hline $\begin{array}{l}\text { Material No impreso (bases de datos, audiocintas, videocintas, } \\
\text { discos compactos, televisores, etc.) }\end{array}$ & $\mathbf{2 7 0}$ & $\mathbf{1 0 0 \%}$ \\
\hline \hline
\end{tabular}

Fuente: Elaboración propia

${ }^{8}$ Los libros de texto del alumno, para el maestro y los avances programáticos así como la guía para el maestro son materiales elaborados de manera impresa y en formato electrónico por la Secretaría de Educación Pública cuya finalidad es asesorar al profesor y al alumno en su práctica escolar mediante unas serie de orientaciones didácticas y de evaluación que contienen sugerencias de actividades de enseñanza-aprendizaje con múltiples alternativas prácticas. 
Para complementar la pregunta anterior, se averiguó también en los docentes acerca de la frecuencia sobre el uso de éstos materiales y el modo en qué prefieren consultarlos (impresa, electrónica, sonora u otra). Los resultados arrojados puntualizaron que en su mayoría los emplearon más de una vez a la semana (77.0\%), un número reducido comentó que hace uso de éstos a diario (2.6\%). (véase tabla 7). Con respecto a su preferencia por utilizarlos 233, (86.2\%), prefirieron optar por el uso del formato electrónico. Solo un número pequeño los consultó de manera impresa (32) (véase figura 2), y en bajos promedios de manera visual (3) y sonoro (2).

Tabla 7. Frecuencia de uso de los materiales

\begin{tabular}{||l||c|c||}
\hline \multicolumn{1}{|c||c}{$\begin{array}{c}\text { Frecuencia de uso de los } \\
\text { materiales }\end{array}$} & $\begin{array}{c}\text { No. de } \\
\text { docentes }\end{array}$ & \% \\
\hline \hline Diario & 7 & 2.6 \\
\hline \hline Una vez a la semana & 34 & 12.6 \\
\hline Más de una vez a la semana & 208 & 77.0 \\
\hline \hline Ocasionalmente & 21 & 7.8 \\
\hline \hline Total & 270 & 100 \\
\hline
\end{tabular}

Fuente: Elaboración propia

Figura 2. Forma en que prefieren consultar la información

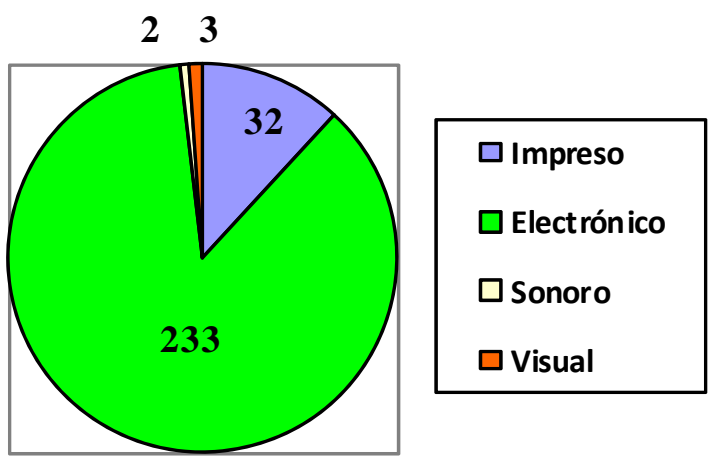

Fuente: Elaboración propia

Una situación que resultó complementaria para la investigación fue conocer el motivo del porque la comunidad de pedagogos prefirió consultar el formato electrónico. Referente a este interés, los profesores indicaron que principalmente lo emplearon debido a su disponibilidad (41.9\%), puesto que en la actualidad se pueden encontrar en Internet diversas fuentes y recursos como los libros de carácter general o materiales que han sido elaborados por la Secretaría de Educación Pública que han representado un gran apoyo en la realización de sus 
actividades académicas y laborales. (véase tabla 8) Aunque también, mostraron que por su accesibilidad (25.5\%), y por su relevancia (20.7\%).

Tabla 8. Motivo por el que prefiere consultar en un formato especifico la información

\begin{tabular}{||l|c|c||}
\hline \multicolumn{1}{|c|}{ Motivo para su consulta } & $\begin{array}{c}\text { No. de } \\
\text { docentes }\end{array}$ & Porcentaje \\
\hline \hline Por su disponibilidad (esté listo por sí se necesita) & 113 & $41.9 \%$ \\
\hline \hline Por su accesibilidad (esté en el lugar adecuado) & 69 & $25.5 \%$ \\
\hline \hline Por su actualidad (sea lo último que ha aparecido) & 17 & $6.3 \%$ \\
\hline \hline Por su oportunidad (esté listo justo en el momento que se necesita) & 3 & $1.1 \%$ \\
\hline \hline Por su pertinencia (trate sobre el asunto que se investiga) & 11 & $4.1 \%$ \\
\hline \hline Por su relevancia (sea importante para la actividad que se realiza) & 56 & $20.7 \%$ \\
\hline \hline Por su calidad (contenga un tamaño, pasta, presentación, etc.) Total & 1 & $0.4 \%$ \\
\hline \hline \multicolumn{1}{|c|}{$\mathbf{2 7 0}$} & $\mathbf{1 0 0 \%}$ \\
\hline
\end{tabular}

Fuente: Elaboración propia

En otro tenor de ideas, también se cuestionó con referencia a su asistencia a los Centros de Maestros por ser considerados como un espacio de asesoría en donde los docentes pueden disponer de sus instalaciones y servicios de biblioteca. Bajo este panorama, se encontró que la gran mayoría acudió poco a los Centros (54.1\%), otros docentes no asistieron (39.6\%), y únicamente algunos (6.3\%), tuvieron una presencia continua. (véase figura 3) Entre las razones que expusieron los docentes que no asistieron a los Centros de Maestros se encontró que principalmente fue por la falta de tiempo (49.5\%), (véase tabla 9), algunos mencionaron que se debió a la distancia en donde se ubican (26.2\%), otros más dijeron que no asistieron porque no tiene los materiales que ellos utilizan (17.8\%), o bien, porque no supieron localizar la información (6.5\%).

Figura 3. Asistencia a los Centros de Maestros

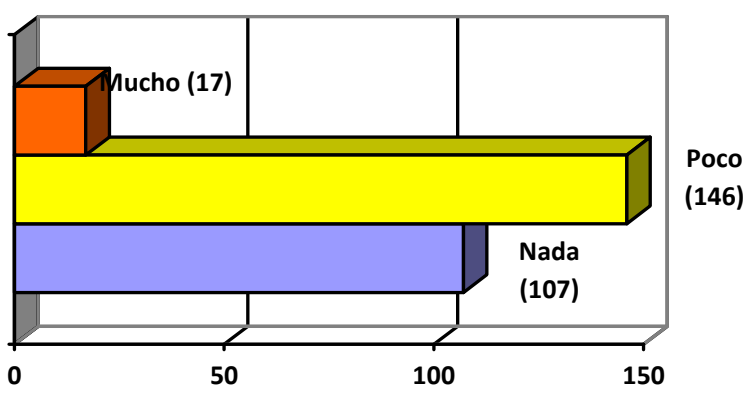

Fuente: Elaboración propia 
Tabla 9. Razón por la que no acude a los Centros de Maestros

\begin{tabular}{|c|c|c|}
\hline Razones & $\begin{array}{c}\text { No. de } \\
\text { docentes }\end{array}$ & $\%$ \\
\hline Me quedan lejos de mi casa o centro de trabajo & 28 & $26.2 \%$ \\
\hline No sé cómo localizar la información & 7 & $6.5 \%$ \\
\hline No tiene los materiales que necesito & 19 & $17.8 \%$ \\
\hline No tengo tiempo & 53 & $49.5 \%$ \\
\hline Total & 107 & $100 \%$ \\
\hline
\end{tabular}

Fuente: Elaboración propia

A razón, de los profesores que asistieron -mucho o poco- (163) (véase tabla 10). Los resultados indicaron que su interés en los Centros de Maestros se debió fundamentalmente a tomar cursos de actualización (58.3\%) que se impartieron dentro de sus instalaciones como un requisito a cubrir para participar en el Programa de Carrera Magisterial. ${ }^{9}$ Por otra parte, también hubo quien asistió para consultar las convocatorias (20.2\%) y solo el $13.6 \%$, a consultar algún material.

Tabla 10. Causa por la que asisten a los Centros de Maestros

\begin{tabular}{||l||c||c||}
\hline \multicolumn{1}{|c||}{$\begin{array}{c}\text { Causa de su asistencia a los Centros de } \\
\text { Maestros }\end{array}$} & $\begin{array}{c}\text { No. de } \\
\text { docentes }\end{array}$ & Porcentaje \\
\hline \hline A consultar algún libro, revista, etc. & 22 & $13.6 \%$ \\
\hline \hline A sacar fotocopias & 5 & $3.1 \%$ \\
\hline \hline A hacer trabajos escolares & 2 & $1.2 \%$ \\
\hline \hline A pedir asesoría sobre un tema & 4 & $2.4 \%$ \\
\hline \hline A consultar las convocatorias & 33 & $20.2 \%$ \\
\hline \hline A asistir a cursos, talleres, etc. & 95 & $58.3 \%$ \\
\hline \hline A formar círculos de estudio & 2 & $1.2 \%$ \\
\hline \hline
\end{tabular}

Fuente: Elaboración propia

Para terminar, a su vez se les cuestionó acerca de la importancia en el desarrollo de diversos cursos que los orienten sobre la manera de cómo buscar y en dónde

${ }^{9}$ Es el programa de incentivos y de evaluación relacionado con la calidad del trabajo educativo de mayor impacto en el país. Comienza su implementación en 1993. Su objetivo fundamental es contribuir a elevar la calidad de la educación básica, reconociendo y estimulando la labor de los mejores profesores a la vez de propiciar la capacitación, actualización y superación profesional mediante su participación en procesos de formación continua que contribuyan a mejorar su desempeño. Para asignar los estímulos salariales, el sistema de evaluación en la actualidad se enfoca en seis diferentes "factores". 
buscar la información. (véase tabla 11) A este respecto, los docentes aludieron lo siguiente, 57 docentes estuvieron a favor y, 18, no lo consideraron necesario.

Tabla 11. Consideran indispensable que se impartan cursos en las bibliotecas o centros de trabajo

\begin{tabular}{|c|c|c|}
\hline $\begin{array}{c}\text { Consideran indispensable } \\
\text { los cursos }\end{array}$ & $\begin{array}{c}\text { No. de } \\
\text { docentes }\end{array}$ & Porcentaje \\
\hline Si & 201 & $74.4 \%$ \\
\hline No & 69 & $25.6 \%$ \\
\hline Total & 270 & $100 \%$ \\
\hline
\end{tabular}

Fuente: Elaboración propia

A continuación se dan a conocer algunas de las razones que expresaron los profesores que respondieron de manera positiva y aquellos que respondieron negativamente.

\section{Si consideran indispensable los cursos}

- no dispongo de mucho tiempo y si es asistido es más rápido

- es tan extenso los diversos títulos y autores que el buscador se pierde

- para una búsqueda más rápida de la información

- para actualizarme y tener una mejor preparación

- es importante utilizar diversas herramientas para informarnos

- para capacitación y actualización

- para recomendarnos títulos o darnos información

- para estar mayor informados y actualizados

- como asistencia en dudas

- crece mucho el acervo cultural

- agilizar y optimizar la búsqueda

- siempre y cuando los temas sean útiles para desarrollarlos en nuestro centro de trabajo

- los requiero para mi práctica docente

- para encontrar más rápido lo que buscamos

- sería más rápido encontrarla y me pueden sugerir lo más actual

- para conocer el uso y contenidos de la misma

- es un apoyo para actualizarse, reforzar e informarse

- es muy importante para tu formación y el desarrollo de estas habilidades en nuestros alumnos

- en ocasiones no sabemos realizar las búsquedas

- para mejorar como profesores

- facilitar la localización de temas específicos

- es sumamente necesario contar con información para mejorar y ampliar nuestros conocimientos

- damos por sentado que sabemos buscar información y organizarla

- la información es diversa y requiere ser buscada en espacios diversos

- existe mucho material disponible muy acorde al nivel, que no se utiliza por no conocerlo

- es indispensable mejorar en nuestra preparación integral educativa

- en la actualidad la tecnología ha superado los niveles de preparación de los docentes

- ayudaría mucho más y así podría apoyar a mis alumnos en adquirir conocimiento

- cada día hay más fuentes de consulta

- para ampliar mi conocimiento y pedirle a los alumnos que asistan 
- encontraríamos rápido lo que buscamos y podrían ampliar nuestra búsqueda (sugerir autores, nuevas ediciones, etc.,)

- para intercambio de experiencias

- para conocer la lógica de archivo

- se abren más opciones de actualización

\section{No consideran indispensable los cursos}

- con un fichero bien organizado sería suficiente

- tengo otros medios para hacerlo

- mejor es ampliar el acervo de libros

- con que organicen los libros y aparezcan en los ficheros o bases de datos es suficiente para buscar

- existen ficheros acerca de los libros y herramientas

- creo que el profesor por su nivel de preparación ya debe de manejar búsqueda informativa

- la diversidad de contenidos y el grado de especificidad con una guía de contenido creo que es suficiente

- todo se puede realizar en casa por Internet

- es más fácil crear un folleto o video para aprender a usar las herramientas

- generalmente encuentro la información que necesito sin ayuda

- posiblemente con un instructivo sería suficiente

- nosotros buscamos en donde se nos facilite

- porque manejo bien lo que necesito

- porque no tengo tiempo

- en la escuela nos brindan estos conocimientos

- porque a través del tiempo uno va adquiriendo experiencia en donde buscar

\section{CONCLUSIONES}

Debido a los resultados obtenidos, es posible afirmar que la comunidad docente de secundaria en México se encuentra representada por las mujeres, hecho que parece tener relación, con la visión tradicional del papel de la mujer en la atención al cuidado de los hijos o bien, a una práctica familiar que se ha heredado de generación a generación dando lugar a que la mujer sea encaminada a ejercer está profesión, además, de que es un trabajo donde no se requiere de una fortaleza física para desempeñar las funciones propias de un docente. De igual forma, hay que precisar que dentro de la profesión docente resalta una doble o triple particularidad, puesto que aparte de predominar las mujeres preponderaron las relaciones de pareja y los dependientes económicos, lo que permite suponer responsabilidades familiares y económicas para la mayoría de las profesoras y profesores que pueden ser factores en diferentes grados para influir en la realización de otras actividades académicas y labores.

Con respecto a su edad, en general se logra determinar que se caracteriza por ser una comunidad adulta que tiene ya varios años en las actividades que desarrolla. Por ello, es importante aludir que dentro de los planteles, existe una ventaja al estar sociabilizando profesoras y profesores con experiencia que consigue ser un referente de gran utilidad para ser retomadas por otros docentes. Además de que son 
profesoras (res) que se encuentran en jornada completa impartiendo clases lo que permitir el intercambio no sólo de experiencias sino también de aprendizajes. Aunque hay que resaltar que por ser profesoras (res) de tiempo completo existen múltiples actividades - pasar asistencia, controlar la disciplina, dar el tema, poner ejercicios, calificar de forma permanente, atender a los padres, preparar sus clases, etcétera- que pueden condicionarlos para llevar a cabo otras actividades relacionadas con su labor académica y desarrollo profesional. En lo referente a su preparación, actualización y superación, las profesoras y profesores cuentan con el nivel de licenciatura y, en la actualidad, no se encuentran cursando algún estudio, lo que guarda relación con su baja asistencia a los Centros de Maestros. Aunque sería necesario profundizar sobre este aspecto para valorar si existe una correlación en cuanto a su preparación y asistencia a los centros.

Por otra parte, y para comprender el comportamiento informativo que caracteriza a la comunidad docente, es necesario mencionar que la introducción de las tecnologías de la información y comunicación a principios de la década de los ochenta y el desarrollo constante de nuevas tecnologías en la recuperación de la información ha hecho que los hábitos de búsqueda en la comunidad de profesores cambien. De este modo, lo que en un principio era un recurso fundamental para el desarrollo de sus actividades de las profesoras y profesores se ha transformado con el avance tecnológico. Hecho que se confirma a través de los resultados arrojados por la comunidad de docentes al afirmar que en la actualidad el Internet es el recurso informativo de mayor importancia. Pero si bien, el Internet hoy en día es el recurso que más emplean las profesoras y profesores para desarrollar sus actividades, la accesibilidad, es la razón que justifica su uso y que estuvo presente en las respuestas obtenidas.

Además de estas evidencias, hay que precisar que dentro de la modalidad general existe una predilección por consultar de manera electrónica los materiales -libros de carácter general- debido a la disponibilidad que representa. Elección que parece natural debido a la incorporación cada vez más de las tecnologías de la información y comunicación en la vida laboral.

También cabe agregar que dentro del comportamiento que manifiestan las profesoras y los profesores se encuentra el uso de los Centros de Maestros. A este respecto, hay que hacer alusión que la presencia de los docentes en éstos recintos es muy baja e inclusive nula, debido a que revelaron que la razón principal de su poca asistencia es el tiempo que tienen para poder realizar otras actividades. Razón por la cual se podría pensar que algunos factores como su estado civil, dependientes económicos, distancia que hay entre su casa o el centro de trabajo a la biblioteca, están ejerciendo presión de alguna manera para que los profesoras (es) no acudan a estos recintos.

Un aspecto más para identificar las características de su comportamiento en la búsqueda de la información de la comunidad se encuentra encaminado hacia el conocimiento con respecto a la importancia de asistirlos mediante la impartición 
de cursos que los apoyen para la búsqueda y obtención de la información. Bajo esta inquietud una minoría no estuvo de acuerdo.

Respecto a la primer hipótesis, que asevera que el procedimiento de búsqueda de información que los profesores han desarrollado se dirige principalmente hacia el uso de los materiales impresos, dejando de lado el empleo de Internet y otros medios electrónicos. Asimismo, existe una mayor tendencia a recurrir hacia los Centros de Maestros que a la comunicación entre colegas. Se prueba de forma negativa, a causa de que el profesor prefiere recurrir al uso de Internet por la accesibilidad que representa y emplear de manera electrónica los libros de carácter general y los libros para el maestro, además que mostró que su asistencia a los Centros de Maestros es baja, y en un segundo lugar, preguntar a sus colegas o, bien, dirigirse al uso de su colección personal.

Por último, a razón de la siguiente hipótesis que sostiene que las fuentes y recursos electrónicos son utilizados con mayor frecuencia por los profesores jóvenes en comparación con aquellos colegas que tienen una edad mayor, se prueba de forma negativa, debido a que la edad no fue un factor que influyera hacia la frecuencia en el uso de las fuentes y recursos electrónicos, al menos dentro de la comunidad docente que se caracteriza por ser una comunidad adulta.

Finalmente, cabe mencionar que esta primera exploración que se ha llevado a cabo en la comunidad docente del nivel secundaria puede representar un interés particular para otros actores que deseen profundizar en algún aspecto. Por lo que resulta evidente considerar la importancia de formar cuadros de profesionales que se dediquen a enriquecer esta línea de investigación, ya que en nuestro país la temática aún sigue siendo incipiente; y más aún, para continuar con la gran batalla de la ignorancia que se tiene sobre la importancia de conocer y aplicar en nuestras comunidades de usuarios investigaciones que permitan contribuir al conocimiento que hasta el momento se tiene.

\section{REFERENCIAS BIBLIOGRÁFICAS}

ABARCA FERNÁNDEZ, Ramón R. (2007). "La epistemología: herramienta para precisar los campos científicos”. Entelequia. Revista Interdisciplinar, no.3, pp.75-83.

ARRIOLA NAVARRETE, O.; BUTRÓN YÁÑEZ，K. (2008). "La cultura bibliotecaria del usuario de bibliotecas académicas: un panorama de su realidad”. Ponencia presentada en el VI Coloquio de Administración y Liderazgo en el Campo Informativo (ALCI).Veracruz, Boca del Río.

CALVA GONZÁLEZ, Juan José. (coord.). (2013). Estudios de usuarios en diferentes comunidades: necesidades de información y comportamiento informativo. México: UNAM; Instituto de Investigaciones Bibliotecológicas y de la Información.

CHORNESS, M. H., RITTENHOUSE, C. H., HEALD, R. C. (1968). Decision 
processes and information needs in education: a field survey. Berkley, California: Far West Laboratory foe Educational Research and Development. GONZÁLEZ TERUEL, Aurora (2005). Los estudios de necesidades y usos de la información: fundamentos y perspectivas actuales. España: Trea,

HAVELOCK, Ronald, G. (1967). Dissemination and translation roles in education and other fields: a comparative analysis. Ann Arbor, Michigan: University of Michigan.

HAVELOCK, Ronald, G. (1969). Planning for innovation: a comparative study of the literature on the dissemination and utilization of scientific knowledge. Ann Arbor, Michigan: University of Michigan.

HERNÁNDEZ SAMPIERI, Roberto; FERNÁNDEZ COLLADO, Carlos y BAPTISTA LUCIO, María del Pilar (2010). Metodología de la investigación. 5a. ed. México: McGraw-Hill.

QUIÑONES MENÉNDEZ, Ma. Rosa (1992). Prospectiva de la tecnología educativa al año 2000: modificación curricular en la educación media básica. México: SEP.

LÓPEZ SUÁREZ, Adolfo; MORALES HERNÁNDEZ, Ignacio; SILVA BELTRÁN, Elvia E. (2005). La estructura del sistema educativo mexicano. México: Universidad Autónoma del Estado de México.

MARTÍNEZ BENCARDINO, Ciro (2002). Estadística y muestreo. 11a. ed. Bogotá: Ecoe Ediciones.

MÉXICO. "Diario Oficial de la Federación de México". (9 febrero, 2012). Secretaría de Educación Pública. Ley General de Educación. Artículo 3. $<$ http://www.dof.gob.mx/>. [Consulta: 11/10/2012]

MEXICO. Instituto Mexicano de la Juventud. <http://www.imjuventud.gob.mx/>. [Consulta: 9/10/2014]

MEXICO. Secretaría de Educación Pública. "Directorio de Escuelas SEP-DF". $<$ http://www2.sepdf.gob.mx/directorio_escuelas/>. [Consulta: 20/01/2014]

MÉXICO. Secretaria de Educación Pública (2010). Modelo curricular para la formación profesional de los maestros de educación básica. Documento interno no publicable de uso exclusivo en el Seminario Académico para la Reforma Curricular de las Escuelas Normales-DGESPE. México: SEP, Subsecretaria de Educación Superior.

MÉXICO. Secretaria de Educación Pública (2011). Programa Nacional de Carrera Magisterial. Lineamientos Generales. México: SEP. SNTE.

MÉXICO. Secretaría de Educación Pública. Nómina de la Plantilla Docente de nivel secundaria correspondiente a la quincena 19, (octubre), 2012.

MÉXICO. Secretaria de Educación Pública. (2012b). "Secretaría de Educación Pública en el D. F. Estadísticas 2011-2012. Secundarias”.

$<$ www.sep.gob.mx/work/models/sep1/Resource/1899/3/images/principales_cifr as_2011_2012.pdf >. [Consulta: 9/03/2013] 
MÉXICO. Secretaría de Educación Pública. Sistema Educativo de los Estados Unidos Mexicanos, principales cifras, ciclo escolar 2000-2001; 2006-2007 y 2011-2012. México: SEP, Dirección General de Planeación y Programación.

RITTENHOUSE, C. H. (1970). Innovation problems and information needs of educational practitioners. Menlo Park: California: Stanford Research Institute.

ROJAS SORIANO, Raúl (2003). Guía para realizar investigaciones sociales. México: Plaza y Valdés.

SANDOVAL FLORES, Etelvina (2008). La trama de la escuela secundaria: institución, relaciones y saberes. México: Plaza y Valdés: UPN.

SANZ CASADO, Elías (1993). La realización de estudios de usuarios: una necesidad urgente. Revista General de Información y Documentación, vol. 3, no.1, pp.154-166.

SUMMERS, E. G. (1974). Information and bibliographic needs in Canadian education. Paper presented at the National Conference on the State of Canadian Bibliography, Vancouver, British Columbia, Canada. 
Apéndice

\section{Cédula de entrevista}

I) Datos Generales

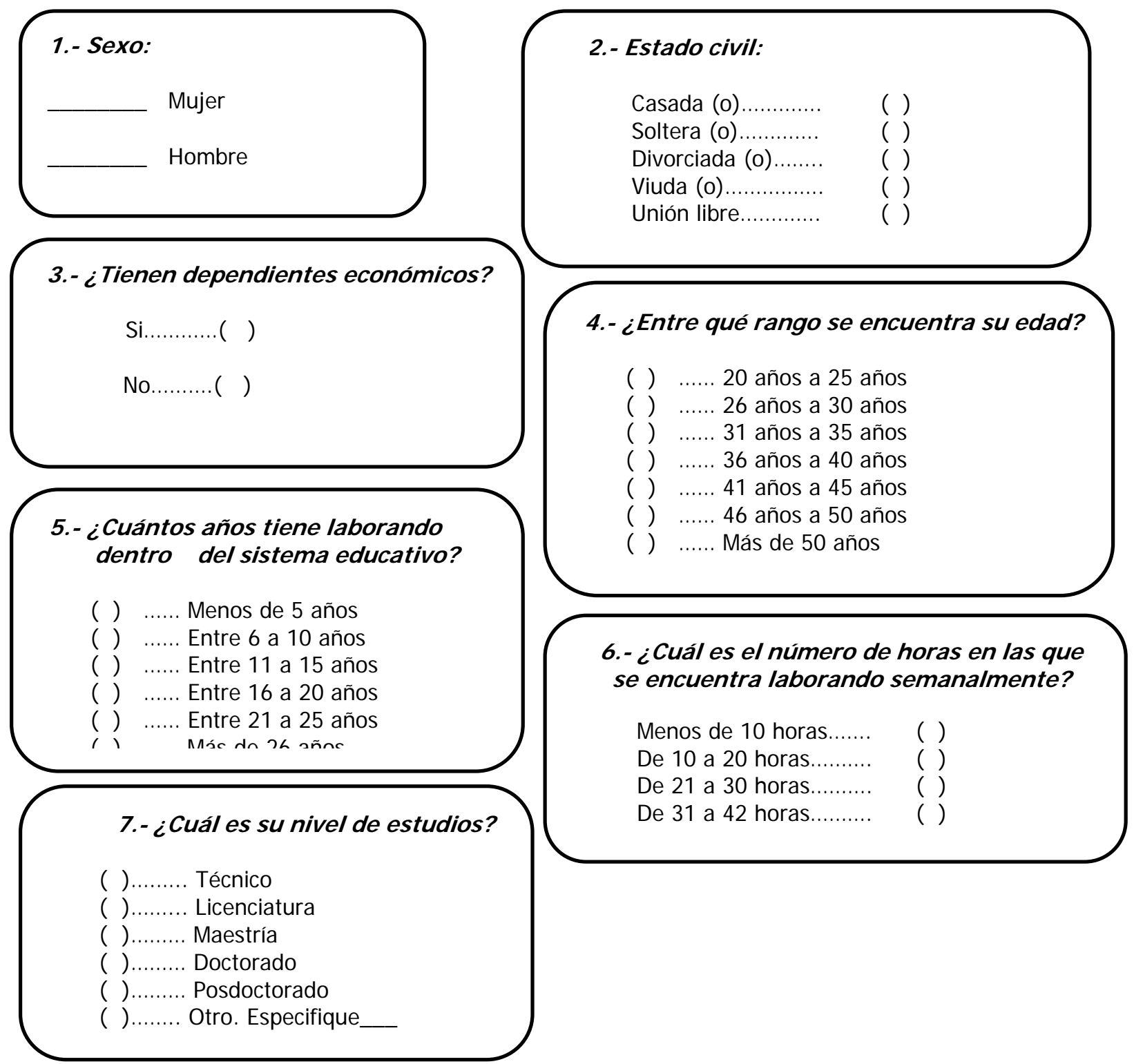




\section{1.- ¿Estudia actualmente?}<smiles>O=[N+]([Si])C1CCCCC1</smiles>

Si su respuesta fue afirmativa.

\section{2.- ¿Qué estudia?}

Curso.

Diplomado.

Especialización

Licenciatura.

Maestría.

nnotoradn

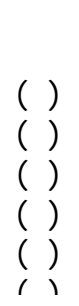

II) Con respecto a su búsqueda de información

\section{8.- Cuando en ud. se origina una necesidad de información, ¿qué acción realiza en un} primer momento?. Por favor indique con el número (1), la que emplea como primera instancia y, con el número (8), la que menos emplea.

Preguntar a sus colegas.

Consultar Internet.

Consultar su colección particular.

Asistir a la biblioteca (Biblioteca escolar, Biblioteca Pública, Centro de Maestros)...

Confiar en su experiencia y conocimientos personales.

Recurrir a eventos (congresos, reuniones, talleres, encuentros, etc.).

Asistir a librerías.

Acudir a puestos de periódicos.

Otra. Especifique

9.- ¿Por qué razón recurre a realizar esta primera acción que indicó con el número (1)?

Por su accesibilidad.

Por su confiabilidad.

Por la rapidez para encontrar la información.

Por su costo. 


\section{0.- ilndique del (1) al (10) los materiales que utiliza con mayor frecuencia para desarrollar sus actividades laborales?. El número (1) indica el material que más emplea y el número (10) el que menos emplea.}

Libros de carácter general........................................................................... ( )

Libros para el maestro

Libros "guía para el maestro".

Libros de apuntes para el maestro

Libros de texto del alumno.

Ficheros de actividades didácticas.

Avances programáticos......

Notas de clase.

Notas de cursos, seminarios, ponencias, talleres, reuniones, congresos etc.....

Material No impreso como: bases de datos, audiocintas, videocintas, discos

compactos, televisores, etc.

Artículos de revistas.

\section{1.- ¿Qué tan frecuente emplea el material que señaló con el número (1)?}

Diario

Una vez a la semana.

Más de una vez a la semana

Ocasionalmente.

Rara vez.

Otra. Especifique

\section{2.- ¿De qué forma prefiere consultar} los materiales?

Impreso.

Electrónico

Sonoro.

Visual

\section{3. ¿ ¿Por qué prefiere consultar estos materiales?}

Por su disponibilidad (esté listo por sí se necesita).

Por su accesibilidad (esté en el lugar adecuado).....

Por su actualidad (sea lo último que ha aparecido)

Por su oportunidad (esté listo justo en el momento que se necesita).............

Por su pertinencia (trate sobre el asunto que se investiga).....

Por su relevancia (sea importante para la actividad que se realiza).

Por su costo.

Por su calidad (que contenga un tamaño, pasta, presentación, etc.).

14.- ¿Qué tan asiduo es a asistir a los Centros de Maestros?

Mucho.............. ( )

Poco................. ( )

Nada................ ( ) 


\section{5. - Si su respuesta fue poco o nada. ¿Por qué no recurre con} frecuencia a los Centros de Maestros?

Porque me quedan lejos de mi casa o centro de trabajo.....

Porque no sé cómo localizar la información....

Porque no tiene los materiales que necesito...

Porque no tengo tiempo.

Otro motivo. Especifique

\section{6.- ¿A qué a asistido a los Centros de Maestros?}

A consultar algún libro, revista, etc.

A sacar fotocopias

A hacer trabajos escolares

A pedir asesoría sobre un tema

A consultar las convocatorias

A asistir a cursos, talleres, etc.

A formar círculos de estudio

Otra. Especifique:

17.- ¿Considera necesario que se desarrollen cursos dentro de las bibliotecas o de las escuelas que le asistan para la búsqueda de la información que necesita?

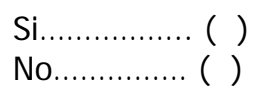

Porque

¡Gracias por su tiempo! 